\title{
Spatio-temporal characterization and control of ultrashort pulses through a multiply scattering medium
}

\author{
A. Tajalli ${ }^{1, a}$, D. J. McCabe ${ }^{1}$, D. R. Austin ${ }^{2,3}$, S. Gigan ${ }^{4}$, I. A. Walmsley ${ }^{2}$, and B. Chatel ${ }^{1}$ \\ 1 CNRS-Université de Toulouse, UPS, Laboratoire Collisions, Agrégats Réactivité, IRSAMC, Toulouse \\ F-31062, France \\ 2 Clarendon Laboratory, Department of Physics, University of Oxford, Oxford OX1 3PU, UK \\ 3 ICFO-Institut de Ciencies Fotoniques, Mediterranean Technology Park, Castelldefels (Barcelona) \\ 08860, Spain \\ 4 Institut Langevin, ESPCI ParisTech, CNRS UMR 7587, Universités Paris 6 \& 7, INSERM, ESPCI, \\ 10 rue Vauquelin, Paris 75005, France
}

\begin{abstract}
Propagation of ultrashort broadband pulses through a multiply scattering media result in complex spatio-temporal speckle pattern. Using spectral pulse shaping, we demonstrate the spatially localized temporal recompression of the output speckle to the Fourier-limit duration.
\end{abstract}

\section{Introduction}

The propagation of a single-frequency laser through a multiply scattering medium produces a fully developed speckle pattern in the spatial domain [1]. This speckle is wavelength dependent, therefore when illuminated by a broadband laser the medium also produces a spectral speckle whose characteristic size is inversely related to the confinement time in the medium, or Thouless time. This corresponds to a severe temporal broadening of the pulse [2]. In the spatial domain, recent experiments have demonstrated that wavefront shaping allows focusing through an opaque medium [3]. For the acoustic and $\mathrm{GHz}$-electromagnetic regimes, time-reversal experiments are the counterpoint to this principle in the time domain [4]. Owing in particular to the inability to measure electric fields directly in the temporal domain at higher frequencies, an optical domain time-reversal experiment remains elusive; yet the ability to measure and shape femtosecond electric fields in the spectral domain nevertheless offers the opportunity of a route to the same goal. Exploiting the coupling of the spatial and spectral modes in the scatterer: recent experiments have also demonstrated a temporal focusing through shaping of the spatial mode $[5,6]$.

Here, we initially demonstrate spatio-temporal characterization of a femtosecond speckle field using Spatially and Spectrally resolved Fourier-transform Interferometry (SSI) [7]. We measure the diffusion properties of the scattering sample [8]. Finally, inspired from time-reversal experiments and using spectral pulse shaping, we demonstrate the spatially localized temporal recompression of the output speckle to the Fourier-limit duration [9]. Our study branches out previous spatial-speckle shaping techniques to the temporal domain and bridges the gap with time-reversal experiments in acoustics and electromagnetism. The ability of recompression of the short pulse behind the sample extends the applications of ultrafast diagnostic techniques including nonlinear microscopy and time-resolved spectroscopy, beyond the range of ballistic photons. Moreover extending the method to 3D measurements would provide an interesting and efficient technique to measure vortices [10].

\footnotetext{
a e-mail: tajalli@irsamc.ups-tlse.fr
} 
(a)

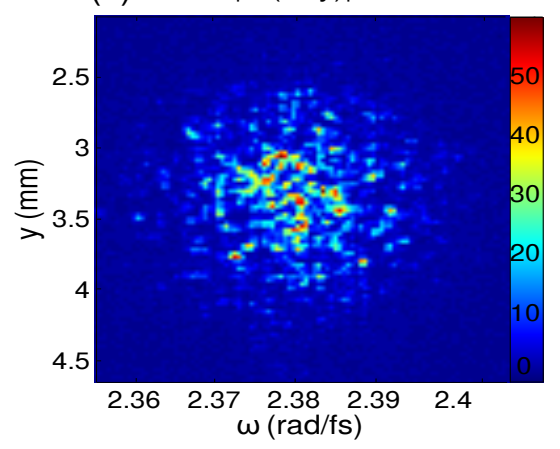

(b)



Fig. 1. Experimental reconstruction of the spatio-spectral electric field of the scattered-transmitted light from a thick scattering sample. (a) Spatio-spectral intensity $I(\omega, y)$ of the speckle. (b) Speckle spatio-spectral phase $\phi(\omega, y)$.

\section{Results and discussion}

In the experiment, an ultrashort pulse is divided into two arms. One arm passes through a pulse shaper and is focussed on a multiply scattering sample. The sample (thick layer of $\mathrm{ZnO}$ powder deposited homogenously on a microscope slide) transforms ultrashort pulse into a complex spatio-temporal speckle pattern. The spatio-temporal speckle is reimaged on the entrance slit of a home-built imaging spectrometer [11]. The other arm acts as a reference and is recombined with an adjustable relative angle and delay at the entrance slit to form a interferometer. The SSI technique performs a relative measurement of the spectral phase between a reference and unknown pulse. The amplitude and phase of the unknown pulse is extracted from raw interferogram via Fourier filtering [12].

Figure 1 shows the reconstructed intensity (a) and wrapped phase (b) of the speckle pattern in the space and frequency domain of the scattering sample. These figures indicate that each point on the speckle pattern possesses random spectral phase and intensity due to the multiply scattering phenomena.

The spatio-temporal transmitted intensity can be reconstructed from the Fourier transform of the spatio-spectral complex electric field along the frequency axis. The average temporal behaviour provides an insight into the properties of the sample. To achieve this, an ensemble-averaged measurement of the temporal profile is taken. The ensemble-average spatio-temporal intensity for 40 measurements is depicted in Fig. 2(a). The spatial integration of the position-averaged intensity yields the averaged transmission intensity, which is plotted in Fig. 2(b). The ensemble average washes out the random fluctuations of the speckle field whilst leaving the underlying statistical properties. By applying a negative exponential fit to the intensity decay, the Thouless time is extracted to be $1046 \mathrm{fs}$.
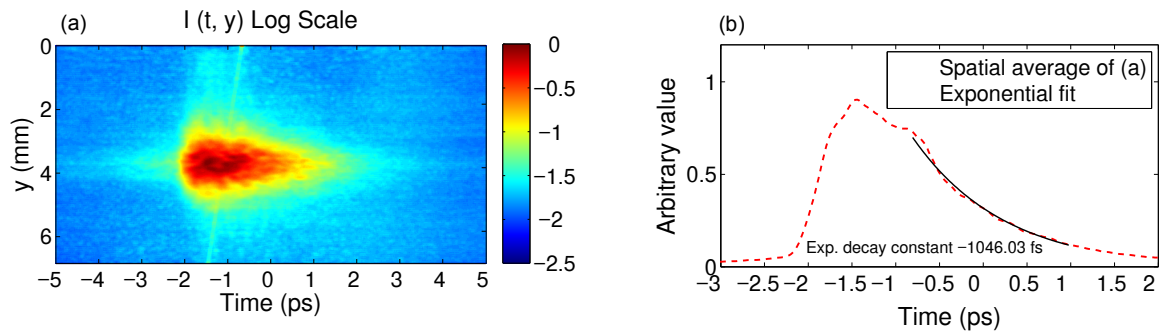

Fig. 2. Position averaged spatio-temporal intensity $I(t, y)$. (a) Ensemble-average of spatio-temporal intensity from forty different positions of the sample. (b) Spatially integrated ensemble-averaged spatio- temporal intensity of (a). An exponential fit of the intensity decay yields a sample decay time of $1046 \mathrm{fs}$. 
Figure 3(a) shows the reconstructed spatially resolved temporal speckle pattern of the scattering sample. This complex structure rises from the interference of the multiply scattered electric field which bears a resemblance to the complex structure of the Fig. 1(a) in spectral domain. Correction of the phase at a chosen point of the pattern using a spectral pulse shaper leads to an intense temporally focussed and spatially localized pulse that emerges from the background of the speckle pattern [See Figure. 3(b)]. The peak temporal intensity is improved by a factor of 15 relative to the average background before compensation. The temporal duration of the focussed pulse is $59 \mathrm{fs}$, which is close to the transform-limited duration of $54 \mathrm{fs}$.
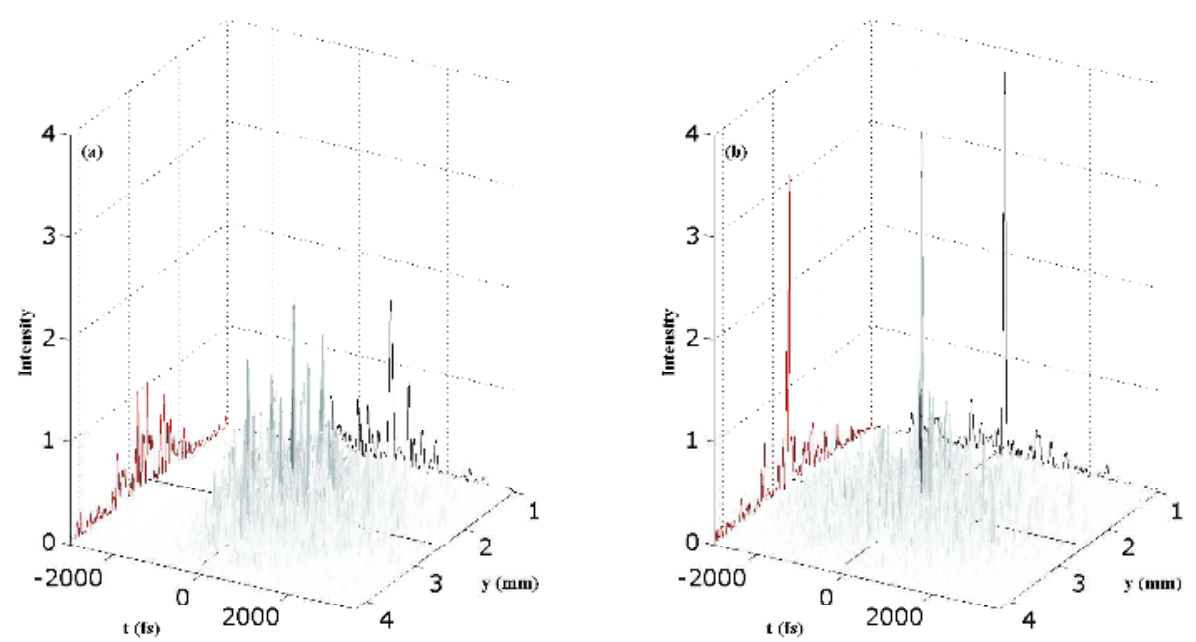

Fig. 3. Reconstructed spatio-temporal intensities (a) before and (b) after compensation of the phase at $y=2.66$ $\mathrm{mm}$. An intense peak emerges from the background with a contrast ratio of 15 . One-dimensional lineouts at the location of this peak are projected onto the walls.

\section{References}

1. J. W. Goodman, J. Opt. Soc. Am, 66, (1976) 1145.

2. M. Tomita, and T. Matsumoto, J. Opt. Soc. Am. B 12, (1995) 170.

3. I. M. Vellekoop, and A. P. Mosk, Opt. Lett. 32, (2007) 2309.

4. M. Fink, Physics Today, 50, (1997) 34.

5. O. Katz, E. Small, Y. Bromberg, and Y. Silberberg, Nature. Photon. 5, (2011) 372.

6. J. Aulbach, B. Gjonaj, P. M. Johnson, A. P. Mosk, and A. Lagendijk, Phys. Rev. Lett. 106, (2011) 103901.

7. T. Tanabe, H. Tanabe, Y. Teramura, and F. Kannari, J. Opt. Soc. Am. B 19, (2002) 2795.

8. A. Tajalli, D. J. McCabe, D. R. Austin, I. A. Walmsley and B. Chatel, J. Opt. Soc. Am. B 29, (2012) 1146

9. D. J. McCabe, A. Tajalli, D. R. Austin, P. Bondareff, I. A. Walmsley, S. Gigan, and B. Chatel, Nature. Commun. 2, (2011) 447.

10. M. V. Berry, J. Phys. A 11, (1978) 27.

11. D. R. Austin, T. Witting, and I. A. Walmsey, Appl. Opt. 48, (2009) 3846.

12. M. Takeda, H. Ina, and S. Kobayashi, J. Opt. Soc. Am. 72, (1982) 156. 